\title{
Cholesterol: a bacterial scarlet letter?
}

Helicobacter pylori, one of the world's most widespread pathogenic human colonizers, has revealed to researchers another of its survival secrets—one that experts hope might help in the search for prophylactic measures against the nefarious microbe.

H. pylori inhabits the stomach lumen of more than half the people on earth, a finding itself so startling that Robin Warren and Barry Marshall received a Nobel Prize in 2005 for discovering the bug and overturning the long-held erroneous dogma that the stomach is too inhospitable for microbial inhabitants. The success of $H$. pylori is largely a result of its stealth; the bacteria are able to persist for decades because of their many techniques for avoiding the host immune system. They are, however, far from innocuous, damaging the stomach with gastic cancer and ulcers.

Although $H$. pylori infection is treatable with antibiotics, even the most effective therapies achieve only $80 \%$ eradication and often require cocktails of two antibiotics and a bismuth compound. This refractory nature of $H$. pylori infection has spurred researchers to study the microbe more closely.

In this context, Thomas Meyer at the
Max Planck Institute for Infection Biology (Berlin, Germany) and his colleagues recently stumbled upon one of $H$. pylori's host-evasion strategies while investigating one of the bacteria's metabolic needs: cholesterol. H. pylori are not able to synthesize cholesterol and must obtain it from their environment-the stomach.

Meyer and coworkers discovered that $H$. pylori extracts cholesterol right from the plasma membrane of the stomach's epithelial cells, but in so doing lit upon the strange fact that mice infected with mouse-adapted H. pylori and fed a $2 \%$ cholesterol diet cleared $95 \%$ of their infection after 25 weeks, a phenomenon not seen in control mice fed a cholesterol-free diet (Nature Med., September).

These results beg the question: does dietary cholesterol improve immune response to $H$. pylori? Indeed, this seems to be the case; the stomachs of cholesterol-fed mice revealed immune-cell infiltration absent in controls. But there's a twist: H. pylori appears to have developed a mechanism for protecting itself from this cholesterol-mediated immune detection. Meyer's team found that $H$. pylori chemically alters most of that cholesterol before incorporating it into its own plasma

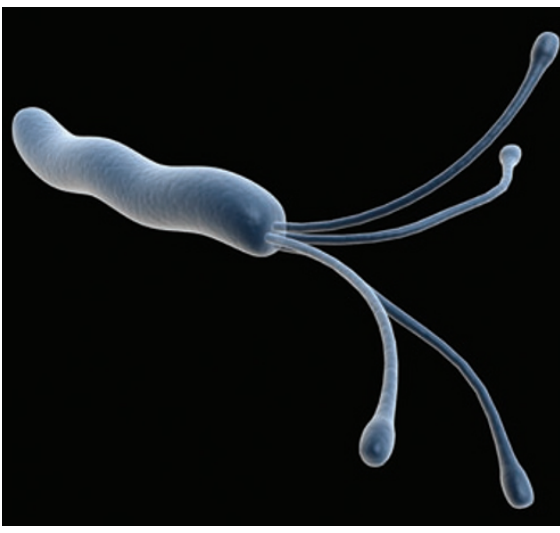

membrane, disguising the molecule from host immune cells. In fact, mice infected with $H$. pylori mutants deficient for the cholesterol-conversion enzyme resolved their infections within 48 hours.

Christian Wunder, one of the paper's first authors, tells Lab Animal that he hopes targeting the cholesterol-conversion enzyme will allow the creation of a "new kind of antibiotic, one that is neither bacteriostatic nor bactericidal by classical definition, but that renders the pathogen accessible to the immune system."

Owen Young

\section{MALARTAL_ARTIETCE_UNMASKED}

Scientists have uncovered a purported mechanism that Plasmodium parasites use to evade mammalian host immune systems. Plasmodium protozoa cause malaria in mammals; the disease afflicts as many as 500 million people each year, roughly one million of whom die annually from its ill effects.

Masters of infectious mayhem, Plasmodium species have proven recalcitrant to study, in part because of their complicated lifecycles. Plasmodium organisms enter their mammalian hosts via bites from Anopheles mosquitoes, after which they progress though various parasitic stages, moving from the bloodstream into liver cells, back to the bloodstream, and finally into red blood cells, where they cause the characteristic fever and chills associated with malaria. One puzzling aspect of the lifecycle is how the liver-stage parasites, called merozoites, are able to avoid the phagocytic immune cells that patrol the liver and bloodstream. Working with $P$. berghei, however, a malarial organism infectious in rodents, Volker Heussler at the Bernhard Nocht Institute for Tropical Medicine (Hamburg, Germany) and his colleagues have elucidated what seems to be one of Plasmodium's mainstay cloak-and-dagger schemes.

According to their new research, published in the 1 September issue of Science, it seems that the merozoites hijack a staple of the immune warning system: apoptosis.

Often called 'programmed cell death', apoptosis is a form of cellular suicide induced by certain conditions, including invasion by foreign organisms. As part of the normal apoptotic process, the cell releases calcium ions that initiate a signaling system to attract pathogen-fighting phagocytes. Heussler's team found evidence that the parasites stall apoptosis in the host cells until the protozoa reach maturity, at which time they signal the host cell to undergo a parasite-induced cell death, escaping into the bloodstream in membrane-bound vesicles termed merosomes. The merosomes are filled with thousands of merozoites and calcium pirated from the now-dying liver cells, leaving those cells unable to signal immune phagocytes and allowing the merozoites to enter the bloodstream undetected.

"We've demonstrated that the translocation of parasites occurs much earlier than originally thought," Heussler tells Lab Animal. "That means treatment against this stage should start much earlier." Moreover, "if we can interfere with the calcium transport to the parasite," he continues, "I predict that the merosomes will be eliminated efficiently by phagocytes."

Owen Young 